\title{
Quantifying the Difference of Uniformly Dispersed and Re-agglomerated Graphene Oxide-Based Cement Pastes on Rheological and Mechanical Properties
}

\author{
Haodao Li , Jingjie Wei, Wujian Long \\ Guangdong Provincial Key Laboratory of Durability for Marine Civil Engineering College of Civil Engineering, Shenzhen University, \\ Shenzhen 518060, China.
}

\begin{abstract}
The state of GO dispersion is closely related to the properties of graphene oxide (GO)-based cement paste. This paper presents the effect of uniformly dispersed and re-agglomerated GO on the rheological and mechanical properties. The results showed that, compared to re-agglomerated GO cement paste, the yield stress and plastic viscosity of uniformly dispersed GO cement paste were higher. Moreover, the compressive and flexural strengths of uniformly dispersed GO pastes were higher than those of re-agglomerated GO pastes. Porosity analysis using mercury intrusion porosimetry showed that the well-dispersed GO can inhibit the formation of large-diameter pores and optimize the pore size distribution better than the re-agglomerated GO.
\end{abstract}

\section{Introduction}

Cement-based composites modified with graphene oxide (GO) additives have been increasingly used in recent years as engineering materials [1]. The use of GO is advantageous since GO is an advanced nanomaterial that exhibits superior performance of GO-incorporated cement pastes. It is clear that this additive does not function solely as a filler, its modifying effect is based on nucleation, followed by aggregation of cement hydration products and formation of a dense mesh structure, ultimately accelerating hydration and improving the early mechanical properties of cement pastes [2]. However, although GO is typically well-dispersed in aqueous solution, an equivalently uniform dispersion in a highly alkaline environment may not be obtained owing to the steric stabilization of GO with charged ions in a cement pore solution [3].

The agglomeration of GO monolithic phases critically affects the properties of GO incorporated cement pastes. It is clear that the abundance of calcium cations plays a significant role in the dispersion of GO in fresh cement pastes [4]. Many studies have been conducted to improve the dispersion of graphene, for example, surface modification of graphene during refining, introduction of foreign molecules and addition of surfactant molecules [5]. An appropriate way for consistent GO dispersion instead of re-agglomeration is mixing with PCE, which can reduce the van der Waals force between GO sheets and disperse them uniformly throughout cement matrix [6].

To compare the differences between uniformly dispersed and re-agglomerated GO, this study systematically investigates the effect of different dispersion states of $\mathrm{GO}$ on the rheological and mechanical properties of cement pastes using two distinct mixing sequences among GO, PCE, and cement.

\section{Materials and experimental procedure}

\subsection{Raw materials}

Type I ordinary Portland cement (OPC), conforming to the requirements of Chinese Standard GB 175[7], was used as the base material in this study. The chemical composition of OPC is provide in Table 1 .

Table 1. Chemical composition of OPC (\% by mass).

\begin{tabular}{cccccccccc}
\hline $\mathrm{CaO}$ & $\mathrm{SiO}_{2}$ & $\mathrm{Al}_{2} \mathrm{O}_{3}$ & $\mathrm{Fe}_{2} \mathrm{O}_{3}$ & $\mathrm{SO}_{3}$ & $\mathrm{MgO}$ & $\mathrm{S}_{\mathrm{r}} \mathrm{O}$ & $\mathrm{Na}_{2} \mathrm{O}$ & $\mathrm{Cl}^{-}$ & LOI \\
\hline 64.65 & 21.88 & 4.49 & 3.45 & 2.44 & 2.36 & 0.90 & 0.51 & 0.010 & 1.31 \\
\hline
\end{tabular}


Graphene oxide was purchased from Sixth Element Ltd., and used probe sonication, a suspension of GO (3 $\mathrm{g} / \mathrm{L})$ was prepared by dispersion the graphite oxide powder in water. The frequency and the time of ultrasound application were $25 \mathrm{~Hz}$ and $2 \mathrm{~h}$, respectively. Polycarboxylate-ether was used as a surfactant to disperse GO in the cement pastes, conforming to the requirement of JG/T 223[8]. As a result, long molecules of the organic polymer wrap around the GO particles and prevent their aggregation by physically blocking them from approaching each other.

\subsection{Test methods}

\subsubsection{Rheological measurements.}

The rheological parameters of the cement paste were measured using an RM 100 touch device manufactured by Lamy Rheology Instruments. During the rheological test, the shear rate was increased from 3 to $240 \mathrm{~s}^{-1}$ over 15 speed intervals. The plastic viscosity $\left(\eta_{p}\right)$ and the shear stress $\left(\tau_{0}\right)$ can be obtained from the slope and the intercept of the Modified Bingham (M-B) model relating the shear stress.

\subsubsection{Mechanical measurements.}

The strengths of the hardened cement pastes were tested according to the procedures of GB/T 17671[9]. The compressive and flexural loading speeds were $2.4 \mathrm{kN} / \mathrm{s}$ and $50 \mathrm{~N} / \mathrm{s}$, respectively.

\subsection{Mix proportions}

A PCE-to-GO mass ratio of 1:1 was chosen based on preliminary results and is consistent with the limited data reported in the literature [10]. The mix proportions of the GO-based cement pastes are given in Table 2 and the only difference in the mixing sequence of the two samples is the pre-mixing of GO with PCE before adding to the cement paste.

\section{Results and discussion}

\subsection{Effect of dispersion state of GOs on the rheological properties}

As can be seen in Figure 1(a), the fresh cement paste containing the re-agglomerated GO (Paste No.1) and uniformly dispersed GO (Paste No.2) show obvious differences in the curve shape but exhibit similar ascending trends prior to solidification, indicating that the two distinct dispersion states of GO in fresh cement pastes evaluated here have a significant effect on the shear stress under different shear rates. Moreover, the shear stress of Paste No.1 is smaller than that of Paste No.2 at the same shear rate, indicating that the reagglomeration of GO may cause a decrease in the shear stress of paste. The possible reasons are the surface area of GO decreases after re-agglomeration, consequently decreasing water absorption and increasing the amount of free water in paste.
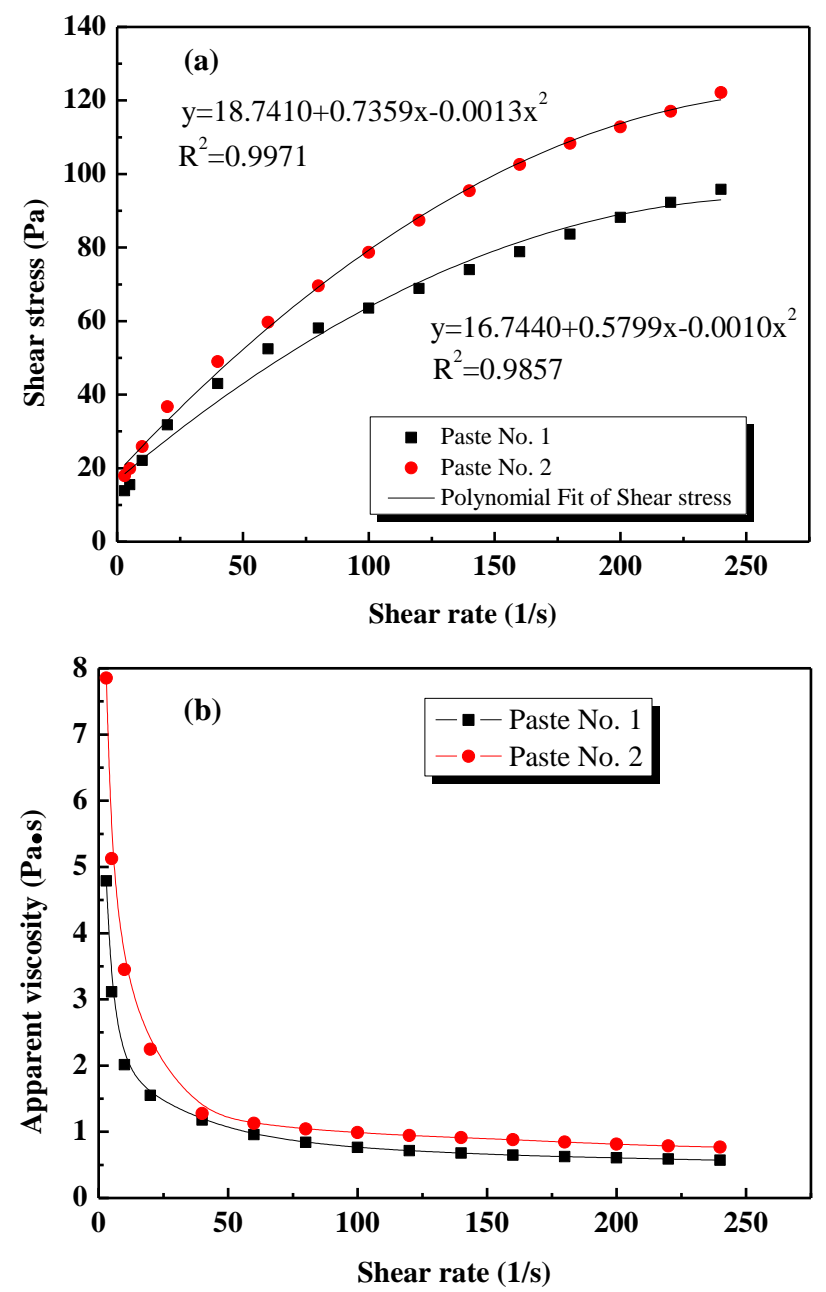

Figure 1. Rheological parameters of GO cement pastes: (a) shear rate vs. shear stress and (b) shear rate vs. apparent viscosity.

Table 2. Mix proportions of cement pastes.

\begin{tabular}{ccccccc}
\hline Mix No. & Cement $(\mathrm{g})$ & Water $(\mathrm{g})$ & W/C & GO $(\mathrm{g})$ & GO/Cement & PCE/GO \\
\hline Paste No. 1 & 3600 & 1440 & 0.4 & 2.16 & $0.06 \%$ & 1.0 \\
Paste No. 2 & 3600 & 1440 & 0.4 & 2.16 & $0.06 \%$ & 1.0 \\
\hline
\end{tabular}


Table 3. Rheological parameters of cement pastes.

\begin{tabular}{ccccc}
\hline Mix No. & Fitting equation & $\tau_{\mathrm{o}}(\mathrm{Pa})$ & $\eta_{\mathrm{p}}(\mathrm{Pa} \cdot \mathrm{s})$ & $\begin{array}{c}\text { Correlation } \\
\text { coefficient }\end{array}$ \\
\hline Paste No. 1 & $y=16.7440+0.5799 x-0.0010 x^{2}$ & 16.7440 & 0.5799 & 0.9857 \\
\hline Paste No. 2 & $y=18.7410+0.7359 x-0.0013 x^{2}$ & 18.7410 & 0.7359 & 0.9971 \\
\hline
\end{tabular}

Figure 1(b) shows that the apparent viscosity of Pastes No.1 and No.2 decrease rapidly with increase in the shear rate and eventually stabilize. Meanwhile, at the same shear rate, the apparent viscosity of Paste No.1 is less than that of Paste No.2 because the flocculated structure in Paste No.1 are destroyed by the applied shear stress, and the re-agglomerated GO is unable to connect any flocculated structure

The yield stress and plastic viscosity of the two cement pastes evaluated in this study are given in Table 3. The results were fitted by the Modified-Bingham (M-B) model, and the correlation coefficient is close to 0.99 , indicating that this fitting model is feasible and appropriate.

\subsection{Effect of dispersion state of GOs on mechanical properties}

Table 4 shows the compressive and the flexural strengths of Pastes No. 1 and No. 2. Both the compressive and the flexural strengths of Paste No. 1 are lower than those of Paste No. 2. The possible reasons include uniform dispersion of GO in Paste No. 2, which facilitates the hydration process of the cement paste, and filling of the inner pores of the cement matrix with GO nanosheets, which increases the density of the cement matrix.

It can be seen from Table 4 that the compressive and flexural strengths of Paste No. 2 at 3, 7 and 28 days were increased by $8 \%, 5 \%$ and $4 \%$ and $27 \%, 26 \%$ and $19 \%$, respectively, when compared to Paste No. 1 . Therefore, it can be concluded that the homogeneous dispersion of GO increases the flexural strength of a paste at an early stage. Clearly, in order to ensure the strength of GO-cement composites, the incorporation of well-dispersed GO into the cement paste must be assured.
The total porosity and the ratio of different pore sizes to the total porosity in Paste No.1 and No.2 at 7 and $28 \mathrm{~d}$ of curing are shown in Fig. 2. According to the influence of different pore sizes on the properties of concrete, the pores can be divided into innocuous pores (less than 0.02 $\mu \mathrm{m}$ in diameter), less harmful pores $(0.02-0.05 \mu \mathrm{m}$ in diameter), harmful pores $(0.05-0.2 \mu \mathrm{m}$ in diameter) and more harmful pores (greater than $0.2 \mu \mathrm{m}$ in diameter). Because both the pore size and pore ratio determine the performance of concrete, increasing the proportion of pores less than $0.05 \mu \mathrm{m}$ in diameter, rather than solely decreasing the proportion of pores greater than $0.05 \mu \mathrm{m}$ in diameter, is more favorable for improving the performance of a concrete, particularly in terms of the durability of the materials.

It can be seen from Figure 7 that the total porosity of Pastes No. 1 and No. 2 are 23.5\%, 17\% and 23\%, 13\% at 7 and 28 days, respectively. The total porosity of a sample trends to reduce as the age increases and the total porosities for different ages of Paste No. 1 are consistently greater than those of Paste No. 2, which fits well with the results of strength testing.

Prior to $28 \mathrm{~d}$, the total porosity of Pastes No. 1 and No. 2 decreases from $26.2 \%$ and $23.9 \%$ to $17 \%$ and $13 \%$, respectively, and the percentage of pores less than 0.05 $\mu \mathrm{m}$ in diameter increases from $63 \%$ and $83.3 \%$ to $84.4 \%$ and $85 \%$, respectively. Therefore, between the ages of 7 and $28 \mathrm{~d}$, the strength of Paste No. 1 increased because of decrease in both the total porosity and the percentage of pores less than $0.05 \mu \mathrm{m}$ in diameter. However, the strength of Paste No. 2 remains higher because the percentage of pores less than $0.05 \mu \mathrm{m}$ in diameter continue to be a significant proportion of the total porosity.

\subsection{Analysis based on MIP}

Table 4. Compressive and flexural strengths of cement pastes (in MPa).

\begin{tabular}{ccccc}
\hline Age (days) & \multicolumn{2}{c}{ Compressive strength (\% increase) } & \multicolumn{2}{c}{ Flexural strength (\% increase) } \\
\hline & Paste No. 1 & Paste No. 2 & Paste No. 1 & Paste No. 2 \\
3 & 43.1 & $46.7(8 \%)$ & 2.2 & $2.8(27 \%)$ \\
7 & 58.5 & $62.0(5 \%)$ & 5.0 & $6.3(26 \%)$ \\
28 & 62.3 & $64.8(4 \%)$ & 7.5 & $8.9(19 \%)$ \\
\hline
\end{tabular}



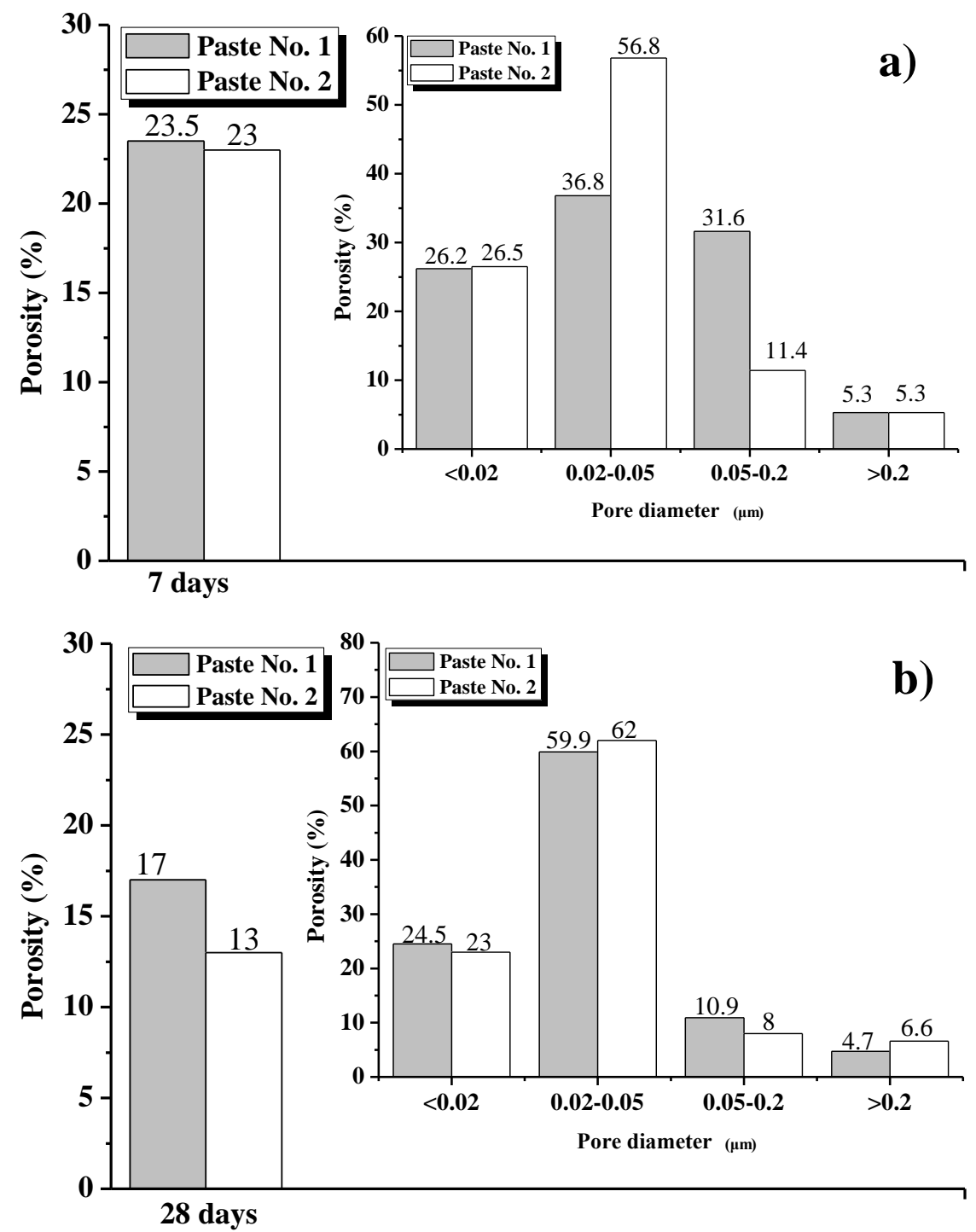

Figure 2. Total porosity of Paste No.1 and No.2 at (a) 7 and (b) $28 \mathrm{~d}$ of curing, and ratio of different pore sizes in range of $<0.02 \mu \mathrm{m}$, $0.02-0.05 \mu \mathrm{m}, 0.05-0.2 \mu \mathrm{m}$, and $>0.2 \mu \mathrm{m}$ to the total porosity.

In summary, hardened cement pastes containing a uniform dispersion of GO have a greater strength than those containing re-agglomerated GO. The hydration process of cement pastes containing uniformly dispersed GO is faster than that of cement pastes containing reagglomerated GO, resulting in a lower cement-based porosity in uniformly distributed pastes at the same age. Additionally, the uniform dispersion of GO is able to increase the ratio of harmless and less-detrimental pores in cement pastes and optimizes the pore size distribution within the matrix, further enhancing the strength of the cement.

\section{Conclusion}

(1) The yield stress and plastic viscosity of Paste No. 2 were higher than those of Paste No. 1. It may conclude that the uniform dispersion of GO increases the water requirement of the paste and GO enhances connections between the flocculated structures of hydration products.

(2) The pores in the cement matrix were filled with GO, restricting the development of cracks. The mechanical properties of Paste No. 2, including compressive and flexural strengths, were higher than those of Paste No. 1.

\section{Acknowledgements}

The authors gratefully acknowledge the financial support provided by the National Natural Science Foundations of China (No. 51778368, No.51578341), and the Shenzhen Overseas High-Caliber Personnel Grant (No. 000095).

\section{References}

1. J. Silvestre, N. Silvestre, J.D. Brito, Eur. J. Environ. Civil Eng. 20, 1-31 (2015) 
2. M. Wang, R. Wang, H. Yao, Z. Wang, S. Zheng, Rsc Adv. 6, 63365-72 (2016)

3. S. Rana, R. Fangueiro, J. Nanomater. 2013, 1-19 (2013).

4. M. Saafi, L. Tang, J. Fung, M. Rahman, J. Liggat, Cem. Concr. Res. 67, 292-99 (2015)

5. A.H. Korayem, N. Tourani, M. Zakertabrizi, A.M. Sabziparvar, W.H. Duan, Constr. Build. Mater. 153, 346-57 (2017).
6. D.G. Gao, Y.J. Ma, Fine Chem. (2015)

7. GB/T175-2007 Chinese national standard (2007)

8. JG/T223-2007 Chinese construction industry standard (2007)

9. GB/T17671-2007 Chinese national standard (2007)

10. M.S. Konsta-Gdoutos, Z.S. Metaxa, S.P. Shah, Cem. Concr. Res. 40, 1052-59 (2016) 